\title{
Downmodulation of dimethyl transferase activity enhances tumor necrosis factor-related apoptosis-inducing ligand-induced apoptosis in prostate cancer cells
}

\author{
CLAUDIO FESTUCCIA ${ }^{1}$, GIOVANNI LUCA GRAVINA ${ }^{1}$, ANNA MARIA D'ALESSANDRO², \\ DANILO MILLIMAGGI ${ }^{1}$, CRISTIANA DI ROCCO ${ }^{5}$, VINCENZA DOLO ${ }^{1}$, \\ ENRICO RICEVUTO $^{1}$, CARLO VICENTINI $^{3}$ and MAURO BOLOGNA ${ }^{4}$
}

\begin{abstract}
Departments of ${ }^{1}$ Experimental Medicine, ${ }^{2}$ Biomedical Sciences and Technologies, ${ }^{3}$ Surgery and ${ }^{4}$ Basic and Applied Biology, University of L'Aquila, I-67100 L'Aquila; ${ }^{5}$ Istituto Dermatopatico dell'Immacolata Roma 3, I-00167 Roma, Italy
\end{abstract}

Received January 16, 2008; Accepted March 11, 2008

DOI: 10.3892/ijo_00000019

\begin{abstract}
One of the major obstacles in curing prostate cancer is the development of drug resistance. It is not only imperative to discover the molecular basis of resistance but also to find therapeutic agents that can disrupt the resistant pathways. Tumor necrosis factor TNF-related apoptosis-inducing ligand TRAIL-like ligands or agonist TRAIL-receptor monoclonal antibodies have entered phase I and II clinical trials with a very limited cytotoxic profile when used systemically in a variety of cancers. Therefore, TRAIL-receptor agonists are new proapoptotic pharmaceutical agents with great potential as new cancer therapeutic agents. Although many cancer cells undergo TRAIL-mediated apoptosis, some are resistant to TRAIL. Therefore, we have been investigating mechanisms to overcome TRAIL resistance in cancer cells so that TRAILassociated compounds can be used effectively in clinical trials. Epigenetic inactivation of proapoptotic genes, or activation of survival signaling, can cause cross-resistance to several antitumor therapies and to immune cytotoxic lymphocytes. We hypothesize that 5-aza-2 deoxycytidine aza-dCR, decitabine may render TRAIL-resistant prostate cancer cells sensitive to caspase-8-mediated apoptosis and may, therefore, be therapeutically efficient. We evaluated the antiproliferative effects of decitabine on the following four prostate cancer cell lines: well-differentiated $\mathrm{AR}$ positive $\mathrm{LnCaP} 53^{+}, \mathrm{PTEN}^{-}$ and 22rv1 p53+ and $\mathrm{PTEN}^{+}$]; poorly-differentiated AR negative PC3 p53-, PTEN ${ }^{-}$and DU145 p53 mutant, PTEN ${ }^{+}$. Here, we provide evidence that treatment with sub-optimal concentrations of decitabine are additive to TRAIL effects in well-differentiated PCa cells whereas the same treatment shows synergistic effects in poorly-differentiated PCa cells
\end{abstract}

Correspondence to: Dr Claudio Festuccia, Department of Experimental Medicine, Via Vetoio, Coppito-2, I-67100 L'Aquila, Italy E-mail: festucci@univaq.it

Key words: prostate cancer, epigenetics, DNA methyltransferase, TRAIL, 5-aza deoxycytidine, decitabine through increased caspase-8 expression, down-modulation of Akt activation and through the expression of certain antiapoptotic molecules including FLIP, PED/PEA-15, survivin and c-IAP-1. Our findings demonstrate that decitabine at relatively low concentrations restores caspase- 8 expression and sensitises resistant PCa cells to TRAIL-induced apoptosis leading to important implications in novel therapeutic strategies targeting defective apoptosis pathways in advanced prostate tumors.

\section{Introduction}

Prostate cancer PCa is one of the most common malignancies among men (1) and in organ-confined tumors, treatments such as radical prostatectomy, radiotherapy and chemotherapy are often effective. This tumor begins as an androgen-dependent, non-invasive disease, which is easily treated with surgery and hormone therapy in early stages (2). However PCa frequently develops into a hormone-independent, highly aggressive and invasive disease (3) and multiple drug resistance often develops in patients with advanced disease.

Promoter $\mathrm{CpG}$ island hypermethylation is an important carcinogenic event in prostate adenocarcinoma. Regardless of tissue type, human cancers have in common both focal $\mathrm{CpG}$ island hypermethylation and global genomic hypomethylation. Transcriptional silencing of different tumor-suppressor genes, associated with aberrant promoter methylation, contributes to tumor initiation and progression and most neoplasms show hypermethylation of one or more genes (4-6).

Lodygin and coworkers (5) as well as Cho and coworkers (6) demonstrated that several $\mathrm{CpG}$ island loci were found in prostate cancer cells and tumors to display cancer-related hypermethylation including RASSF1A, GSTP1, RARB, TNFRSF10C, APC, BCL2, MDR1, ASC, TIG1, RBP1, COX2, THBS1, TNFRSF10D, CD44, p16, and RUNX3.

Demethylating agents, such as azacytidine and its derivative deoxycytidine, have been shown to prevent tumorigenesis in the TRAMP mouse model of PCa (10) and, therefore, could be appealing to molecular targeted therapies (7-9). In addition, the expression of cell death receptors, which usually modulates the number of cells present in a given tissue, is often deregulated 
by transcriptional silencing of downstream molecules. The modulation of tumor growth has also been associated both to the activity of cytotoxic lymphocytes, that kill target cells by various mechanisms including perforin/granzymes and the TNF- $\alpha$ superfamily that induces apoptosis in tumor cells (11). Tumors that develop anti-apoptotic mechanisms can also develop cross-resistance to cytotoxic immune lymphocytes $(12,13)$. The molecular mechanisms that govern anti-apoptotic resistance in cancer cells are numerous and vary from one type of tumor to another. Recent studies suggest that apoptosis resistance, by blockade of the Fas apoptotic pathway, may play an important role in tumor progression in several malignancies (14-16). The TNF-related apoptosis-inducing ligand TRAIL, newest member of the TNF- $\alpha$ family, selectively induces apoptosis through the DR4 and DR5 death receptors. Therefore, TRAIL has attracted much attention as a promising agent for the development for cancer therapy. Unfortunately, cancer cells develop resistance to TRAIL-induced apoptosis through different pathways, including phosphatidylinositol-3-kinase PI3-K/Akt signaling pathway $(17,18)$. Elevated AKT levels in PTEN negative LNCaP cells have been shown to be a major cause of resistance to TRAIL-induced apoptosis (19-21). Agents that could sensitise resistant cancer cells to TRAIL might be particularly important for developing combination regimens that can increase the overall therapeutic efficacy of TRAIL $(22,23)$.

Multiple genes control the apoptotic pathway in order to restrain inadequate cell proliferation and a defect in the signaling mechanism gives the cancer cells an added survival advantage leading to tumor initiation, progression and even drug resistance. Apoptosis is mediated by a family of cystine proteases called caspases. Caspases exist as latent pro-enzymes and are activated by proteolytic cleavage. Several key genes involved in apoptosis have been shown to be the target of epigenetic changes. The death receptor pathway involves the engagement of the death receptors and the recruitment of the adaptor protein FADD and procaspase- 8 to form a complex known as the death-inducing signaling complex. Active caspase- 8 can directly activate caspase- 3 , whose activity is often regulated even by the abundance of apoptosis inhibitors. Caspase- 8 has previously been reported to be frequently inactivated by epigenetic silencing in many tumors including PCa (22). Although TRAIL is a potentially excellent selective therapeutic agent with minimal toxic side-effects, PCa cells develop resistance to it $(24,25)$. We hypothesize that 5 -aza-2 deoxycytidine aza-dCR, decitabine may render TRAILresistant prostate cancer cells sensitive to caspase-8-mediated apoptosis and may, therefore, be therapeutically efficient. Here, we provide evidence that treatment with sub-optimal concentrations of decitabine induces caspase-8 re-expression in poorly-differentiated $\mathrm{PCa}$ cells. Consequently, activation of caspase- 8 and downstream caspases, upon addition of human recombinant soluble TRAIL, was restored by pretreatment with decitabine.

\section{Materials and methods}

Reagents and cells. All tissue culture material was purchased from Hyclone (Cramlington, NE, USA). Plasticware was purchased from Nunc (Roskilde, Denmark). Antibodies, when not otherwise specified, were purchased from Santa Cruz (Santa Cruz, CA, USA). Anti-DNMT1 clone 60 B1220; Biocarta (Hamburg, Germany), -DNMT3a clone 64B 1446; Biocarta, and -DNMT3b clone 52A 1018, Biocarta were used to demonstrate DNMT inhibition. DNA methyltransferase activity was evaluated in nuclear cell extracts using a colorimetric EpiQuik DNA methyltransferase Activity/Inhibitor Assay Kit BioVision (Mountain View, CA, USA). Goat antiTRAIL-receptor antibodies R\&D Systems (Minneapolis, MN). TRAIL KillerTRAIL was purchased from Alexis Biochemicals (San Diego, CA, USA). Akt kinase activity was tested using a non-radio-active assay kit purchased from Stressgene Bioreagents (Victoria, BC, Canada) on cell lysates after partial purification on a MonoQ exchange column as recommended by the supplier. The Caspase- 9 colorimetric kit assay was purchased from Biomol International (L.P Plymouth Meeting, PA, USA). TiterTACS in situ 96-well apoptosis detection kit was purchased from Trevigen (Gaitherburg, MD, USA). 5-Aza-deoxycytidine Aza/dCR, decitabine was purchased from Sigma Aldrich (St. Louis, MO, USA), dissolved in DMSO $100 \mathrm{mmol} / \mathrm{l}$, and aliquots were stored at $-20^{\circ} \mathrm{C}$.

Cell lines. We used LnCaP, 22rv1, PC3 and DU145 human $\mathrm{PCa}$ cell lines which were obtained from the American Tissue Culture Collection ATCC (Rockville, MD, USA).

Growth assays. Cells were seeded at a density of $2 \times 10^{4}$ cells per dish in 50-mm Petri dishes. Cells were left to attach and grow in 5\% FCS DMEM for $24 \mathrm{~h}$. After this time, cells were maintained in different culture conditions. Morphological controls were performed every day with an inverted phasecontrast photomicroscope Nikon Diaphot, Tokyo, Japan, before cell trypsinisation and counting. Cells trypsinised and resuspended in $20 \mathrm{ml}$ of saline, were counted by a haemocytometer every $24 \mathrm{~h}$ (LabRecyclers, Gaithersburg, MD, USA) and 5 independent counts were performed for each dish. All experiments were conducted in triplicate. In order to calculate the inhibitory concentrations at $50 \% \mathrm{IC}_{50}$ of aza$\mathrm{CR}, 2,500$ cells were cultured in 96-well plate for $96 \mathrm{~h}$. After adhesion $16 \mathrm{~h}$, cells were grown as described. After $96 \mathrm{~h}$ the cells were exposed for $4 \mathrm{~h}$ to thyazol blue MTS; Promega (Madison, WI, USA). The 96-well culture plate were then placed on a microplate shaker for $5 \mathrm{~min}$ and the absorbance of the converted dye was measured at the wavelength of $490 \mathrm{~nm}$ using a Bio-Rad multiscan plate reader Bio-Rad (Richmond, CA, USA). Inhibition curves were drawn by means of values obtained by OD percentages versus a control for each concentration. $\mathrm{IC}_{50}$ values were calculated by the GraFit method Erithacus Software Ltd. (Staines, UK) considering the slopes of inhibition curves obtained for each group of tests.

The data obtained from the growth inhibition assay of the co-treatments were analysed using the Combination Index CI that describes the interaction between two mutually nonexclusive drugs i.e. two drugs that have different or independent mechanisms of action. When $\mathrm{CI}<1$ drugs are synergistic, when $\mathrm{CI}=1$ drugs are additive and when $\mathrm{CI}>1$ drugs are antagonistic. The CI is calculated from the following equation: 


$$
C I=\frac{D_{1}}{D_{X 1}}+\frac{D_{2}}{D_{X 2}}+\frac{D_{1} D_{2}}{D_{X 1} D_{X 2}}
$$

A

where $D_{x 1}$ and $D_{x 2}$ are the doses of drug 1 and 2 which are required to inhibit growth by $x \%$.

FACScan analysis cell cycle and apoptosis analysis. Cells to be used for DNA demethylation experiments were grown in the presence of 0.1 to $10 \mu \mathrm{mol} / 1 \mathrm{decitabine}$ for $96 \mathrm{~h}$. Decitabine-containing medium was replaced daily. Cells were treated with TRAIL in complete medium for apoptosis induction. Cell viability was then analyzed by CellTiter 96 assay Promega and adherent cells were trypsinised, pooled with the culture supernatant containing the apoptotic cells already detached from the dish and centrifuged. Cells $\left(1 \times 10^{6}\right)$ were washed in PBS and fixed for $30 \mathrm{~min}$ by the addition of $1 \mathrm{ml}$ of $70 \%$ ethanol. After $30 \mathrm{~min}$, the cells were pelleted by centrifugation $720 \mathrm{~g} ; 5 \mathrm{~min}$, and resuspended in $1 \mathrm{ml}$ of DNA staining solution PBS containing $200 \mathrm{mg} / \mathrm{ml}$ RNase A, $20 \mathrm{mg} / \mathrm{ml}$ propidium iodide plus $0.1 \%$ Triton $\mathrm{X}-100$ and stained by incubation at room temperature for $60 \mathrm{~min}$. All cells were then measured on a FACScan flow cytometer Becton-Dickinson (UK) and analysed using CellQuest software (Becton-Dickinson). Apoptotic cells were detected by a quantifiable peak in sub-G1 phase corresponding to the red fluorescence light emitted by subdiploid nuclei of cells.

Western blot analysis. Cells were washed with cold PBS and immediately lysed with $1 \mathrm{ml}$ of lysis buffer $50 \mathrm{mM}$ HEPES, pH 7.5, $150 \mathrm{mM} \mathrm{NaCl}, 10 \%$ glycerol, $1 \%$ Triton X-100, $1 \mathrm{mM}$ EDTA, $1 \mathrm{mM}$ EGTA, $50 \mathrm{mM} \mathrm{NaF}, 1 \mathrm{mM}$ sodium orthovanadate, $30 \mathrm{mM}$ p-nitrophenyl phosphate, $10 \mathrm{mM}$ sodium pyrophosphate, $1 \mathrm{mM}$ phenylmethylsulfonyl fluoride, $10 \mu \mathrm{g} / \mathrm{ml}$ aprotinin and $10 \mu \mathrm{g} / \mathrm{ml}$ leupeptin. Lysates were electrophoresed in $7 \%$ SDS-PAGE, and separated proteins transferred to nitrocellulose and probed with the appropriate antibodies using the conditions recommended by the antibody suppliers.

Statistical analyses. Data are expressed as the mean + SEM of at least three independent experiments. Statistical analysis was performed using an unpaired Student's t-test.

\section{Results}

It has been demonstrated that decitabine is able to reduce DNA methylation after $24 \mathrm{~h}$ of treatment. Thus a single $24 \mathrm{~h}$ pulse of $0.5-20.0 \mu \mathrm{M}$ decitabine aza-dCR was administered

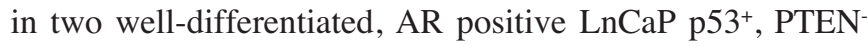
and $22 \mathrm{rv} 1 \mathrm{p} 53^{+}$and $\mathrm{PTEN}^{+}$and two poorly-differentiated AR negative PC3 p53-, PTEN- and DU145 p53 mutant, $\mathrm{PTEN}^{+} \mathrm{PCa}$ cell lines. To measure the proliferative response to aza-dCR, we chose to analyze mitochondria function by MTT absorbance since it measures both cytostatic and cytotoxic responses and since it provides an accurate overall measure of drug sensitivity. Exposure to decitabine resulted in a dose-dependent growth rate reduction as shown in Fig. 1A. A single 24-h pulse of decitabine, followed by $72 \mathrm{~h}$ of culture in drug-free medium, induced $50 \%$ growth inhibition in cells at a concentration $\mathrm{IC}_{50}$ ranging between 0.5 and $25.0 \mu \mathrm{M}$.

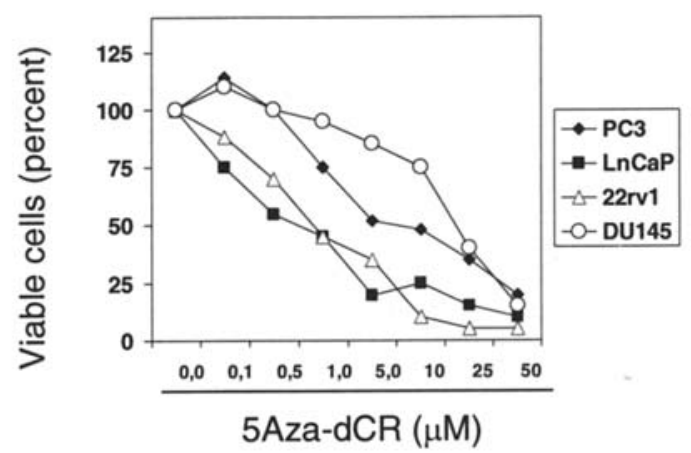

B

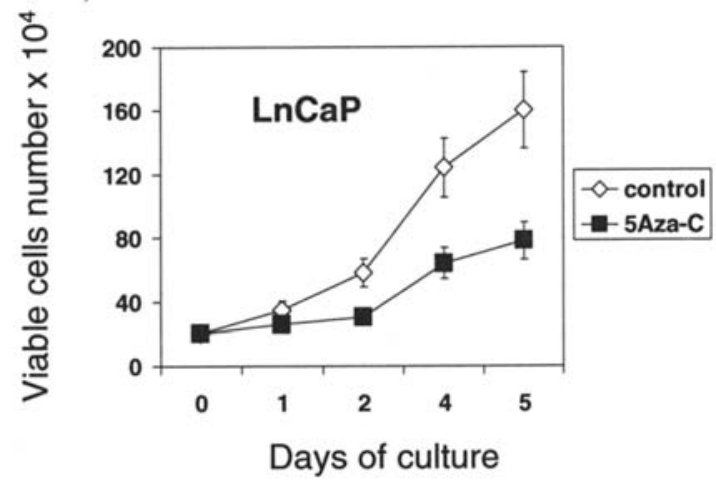

C

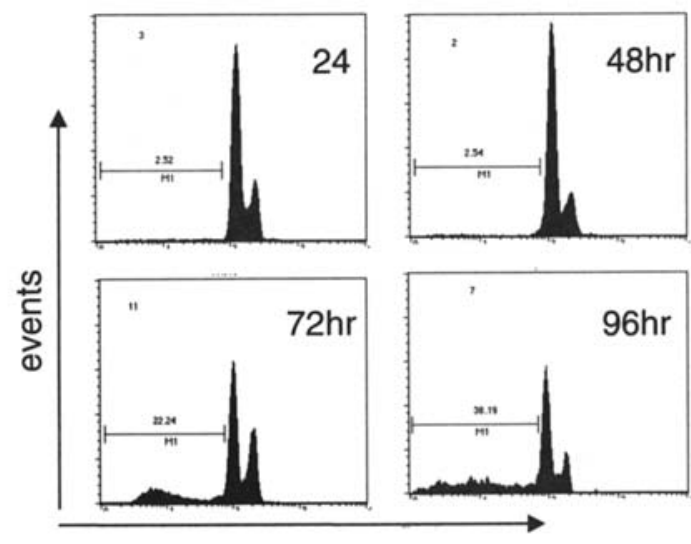

Fluorescence

Figure 1. A, Growth response to aza-CR treatment in androgen-sensitive LNCaP and 22rv1 and in androgen insensitive PC3 and DU145 cells. LnCaP and 22rv1 cells show a higher antiproliferative acute effect when compared to undifferentiated PC3 and DU145 cells. B, Growth curves of LnCaP cells treated or not with $1 \mu \mathrm{M}$ decitabine. C, LnCaP cells treated with $1.0 \mu \mathrm{M}$ decitabine for $24 \mathrm{~h}$ time 0 followed by 24,48 and $72 \mathrm{~h}$ in drug-free medium were induced to trigger apoptotic events in a time-dependent manner.

The effects were higher in 22rv1 $\mathrm{IC}_{50}=0.5 \mu \mathrm{M}$ and $\mathrm{LnCaP}$ $\mathrm{IC}_{50}=1.0 \mu \mathrm{M}$ when compared to $\mathrm{PC} 3 \mathrm{IC}_{50}=5.0 \mu \mathrm{M}$ and DU145 $\mathrm{IC}_{50}=25 \mu \mathrm{M}$ cells. Fig. 1B shows the proliferation curve of LnCaP cells cultured in the presence of $1.0 \mu \mathrm{M}$ decitabine. Cell cycle distribution analysis showed a doseand time-dependent increment of cell proportion in the G0/G1 phase in highly sensitive LnCaP and 22rv1 cells Table I. Furthermore, these cells showed an evident doseand time-dependent increase of apoptotic cells. Fig. 1C shows the FCAScan analysis in LnCaP cells at 24, 48, 72 and 
Table I. Cell cycle distribution in 22rv1, LnCaP, PC3 and DU145 PCa cell lines after a 48-h treatment after a 24-h single pulse of decitabine.

\begin{tabular}{|c|c|c|c|c|c|c|c|c|c|c|c|c|c|c|c|c|c|c|c|c|}
\hline & & & & & & & & & tabin & $(\mu \mathrm{M})$ & & & & & & & & & & \\
\hline & & & $22 \mathrm{rv} 1$ & & & & & $\mathrm{nCa}$ & & & & & $\mathrm{U} 14$ & & & & & PC3 & & \\
\hline & 0 & 1 & 5 & 10 & 25 & 0 & 1 & 5 & 10 & 25 & 0 & 1 & 5 & 10 & 25 & 0 & 1 & 5 & 10 & 25 \\
\hline Apoptosis & $<1$ & 15 & 27 & 20 & 45 & $<1$ & 7 & 25 & 38 & 45 & $<1$ & $<1$ & $<1$ & $<1$ & 5 & $<1$ & $<1$ & $<1$ & 10 & 20 \\
\hline $\mathrm{C} 0 / \mathrm{G} 1$ & 54 & 63 & 76 & 81 & 92 & 64 & 71 & 79 & 78 & 93 & 53 & 55 & 52 & 52 & 47 & 64 & 64 & 55 & 55 & 50 \\
\hline $\mathrm{S}$ & 25 & 22 & 13 & 13 & 7 & 16 & 19 & 11 & 11 & 2 & 27 & 25 & 15 & 7 & $<1$ & 24 & 22 & 25 & 20 & 10 \\
\hline $\mathrm{G} 2 / \mathrm{M}$ & 20 & 15 & 11 & 6 & $<1$ & 20 & 10 & 10 & 6 & 5 & 20 & 20 & 33 & 41 & 53 & 12 & 14 & 20 & 25 & 40 \\
\hline
\end{tabular}

A

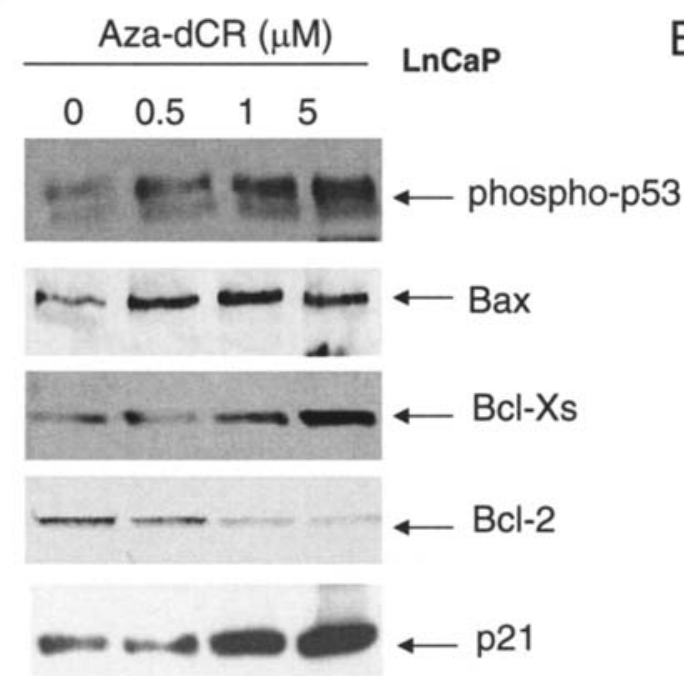

B
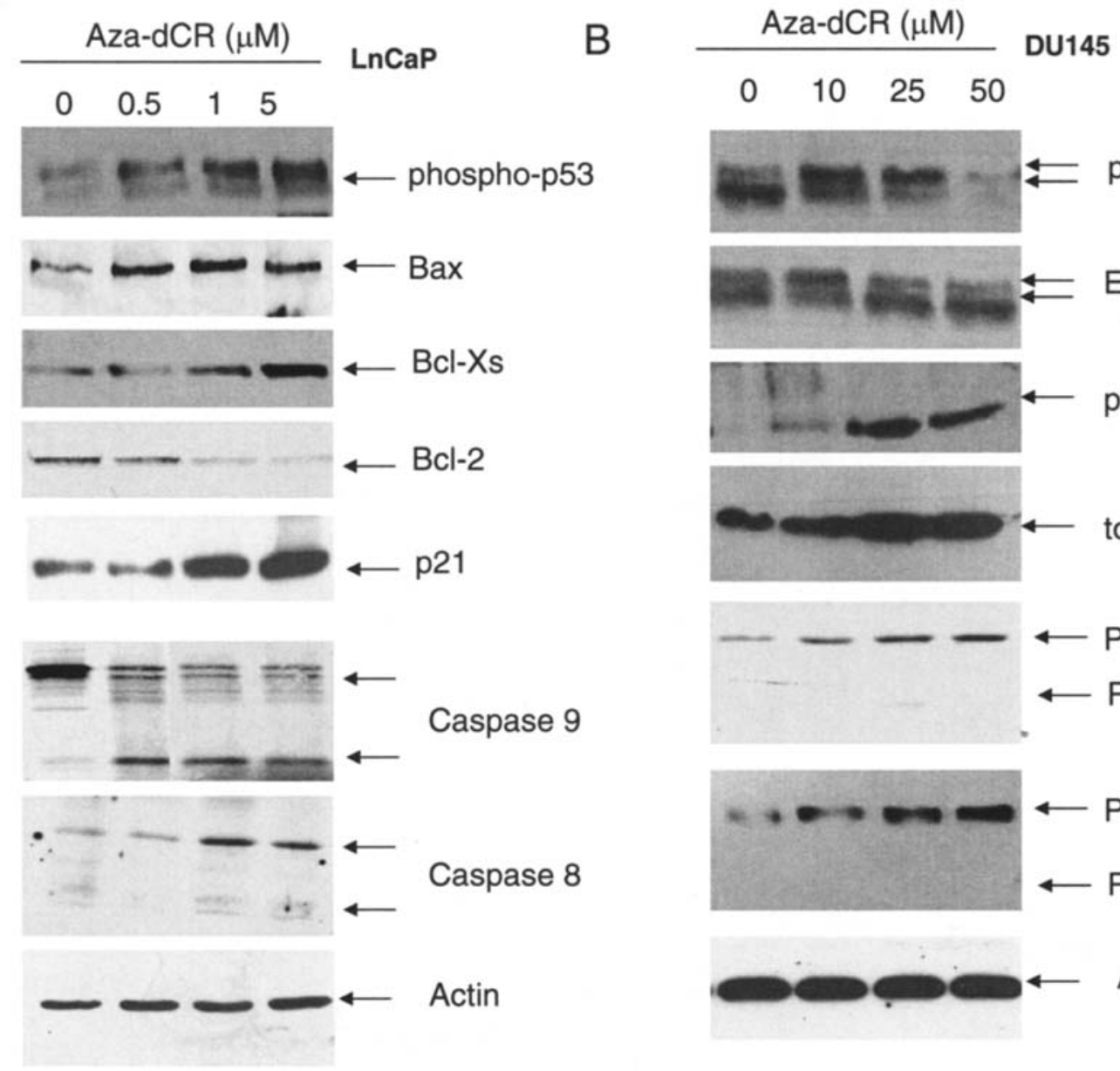

Actin

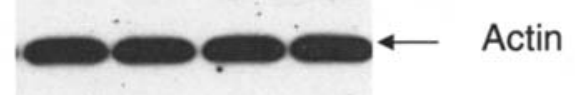

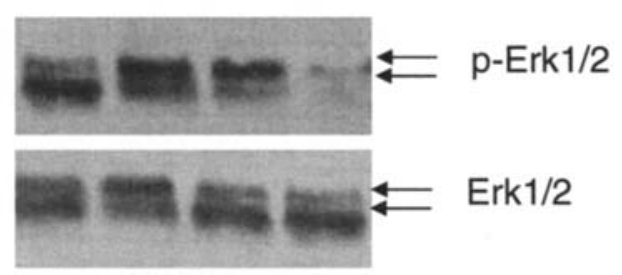
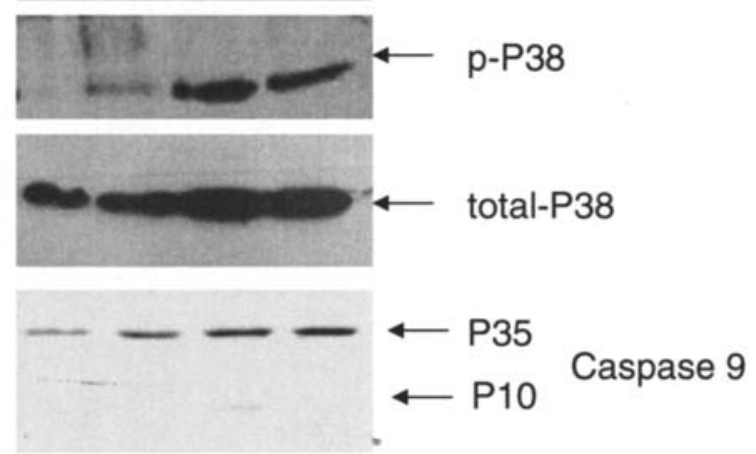

Caspase 9

Caspase 8

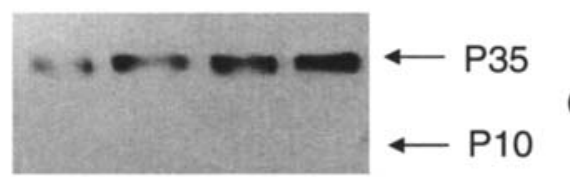

Caspase 8

Figure 2. A, Molecular events involved in decitabine-mediated LnCaP apoptosis. Western blot analysis on the expression of pro-apoptotic and anti-apoptotic elements in LnCaP cells treated with $0.5,1.0$ and $5.0 \mu \mathrm{M}$ decitabine corresponding to $\mathrm{IC}_{20}, \mathrm{IC}_{50}$ and $\mathrm{IC}_{80}$, respectively. B, Modulation of $\mathrm{MAPK}$ pathways in DU145 treated with 10,25 and $50 \mu \mathrm{M}$ decitabine, corresponding to $\mathrm{IC}_{20}, \mathrm{IC}_{50}$ and $\mathrm{IC}_{80}$, respectively. Each lane was loaded with $40 \mu \mathrm{g}$ of proteins. This experiment is representative of three individual experiments.

$96 \mathrm{~h}$ after the single pulse of $24 \mathrm{~h}$ time 0 followed by 24,48 and $72 \mathrm{~h}$ in drug-free medium showing the time-dependent apoptosis induced by decitabine. In PC 3 and DU145 cells, the treatment with decitabine determined very little PC3 or no apoptosis DU145 at all, with a dose- and time-dependent cell cycle increment of cells in $\mathrm{G} 2 / \mathrm{M}$ phase (Table I). We analyzed the molecular mediators of the apoptotic process in $\mathrm{LnCaP}$ and 22rv1 cells which are p53 wt and decitabine responders. We observed reduced $\mathrm{Bcl}-2$ levels with increased levels of phosphorylated p53. This was associated with increased Bax, Bcl-Xs and p21 levels. In parallel, caspase-9 and - 8 were cleaved. Fig. 2A shows these effects in $\mathrm{LnCaP}$ cells at low doses $0.5,1.0$ and $5.0 \mu \mathrm{M}$ of decitabine, corresponding to $\mathrm{IC}_{20}, \mathrm{IC}_{50}$ and $\mathrm{IC}_{80}$ values for this cell line. Similar dose- 

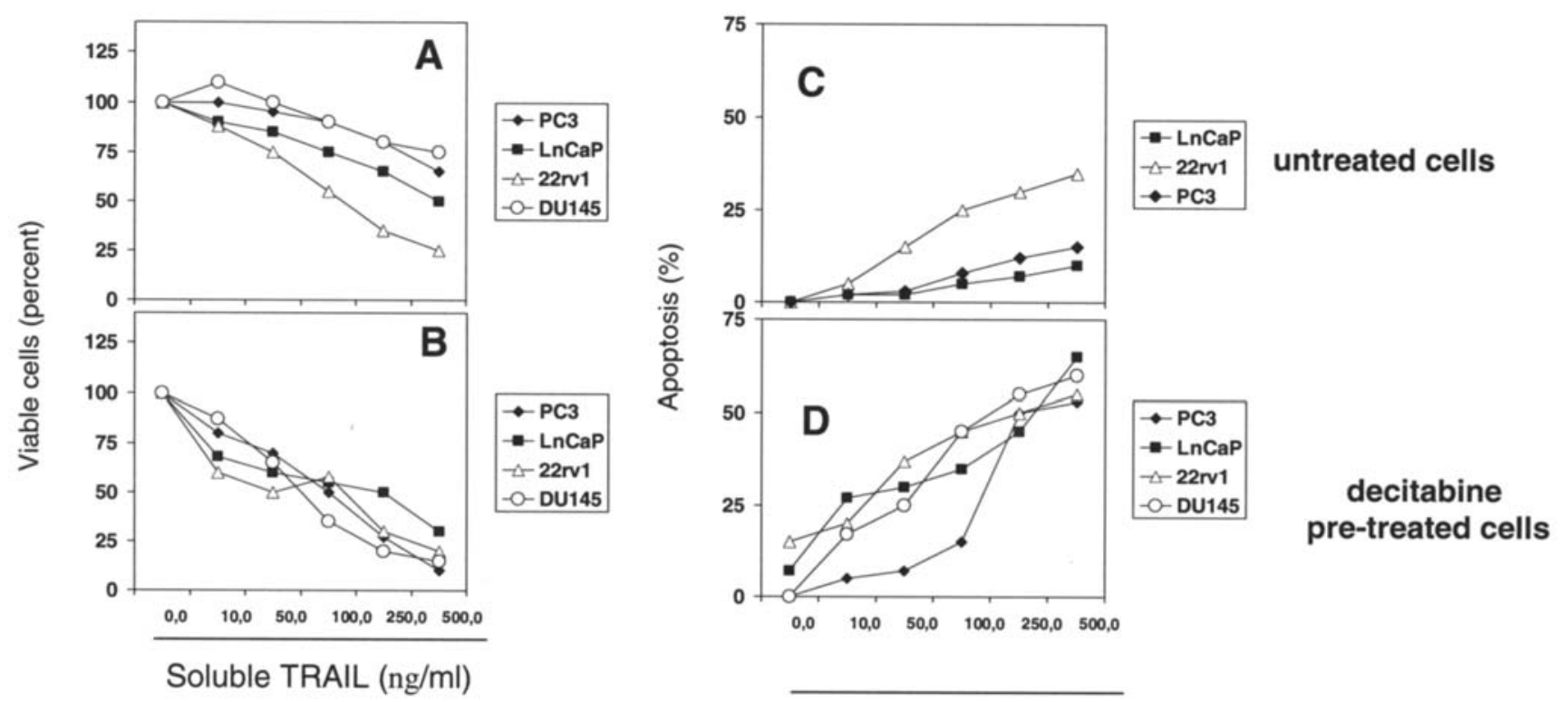

Figure 3. A and B, Comparisons amongst antiproliferative effects to TRAIL with (B) or without (A) $1.0 \mu \mathrm{M}$ decitabine. C and D, Comparisons of apoptotic effects mediated by TRAIL with (A) or without (B) $1.0 \mu \mathrm{M}$ decitabine.

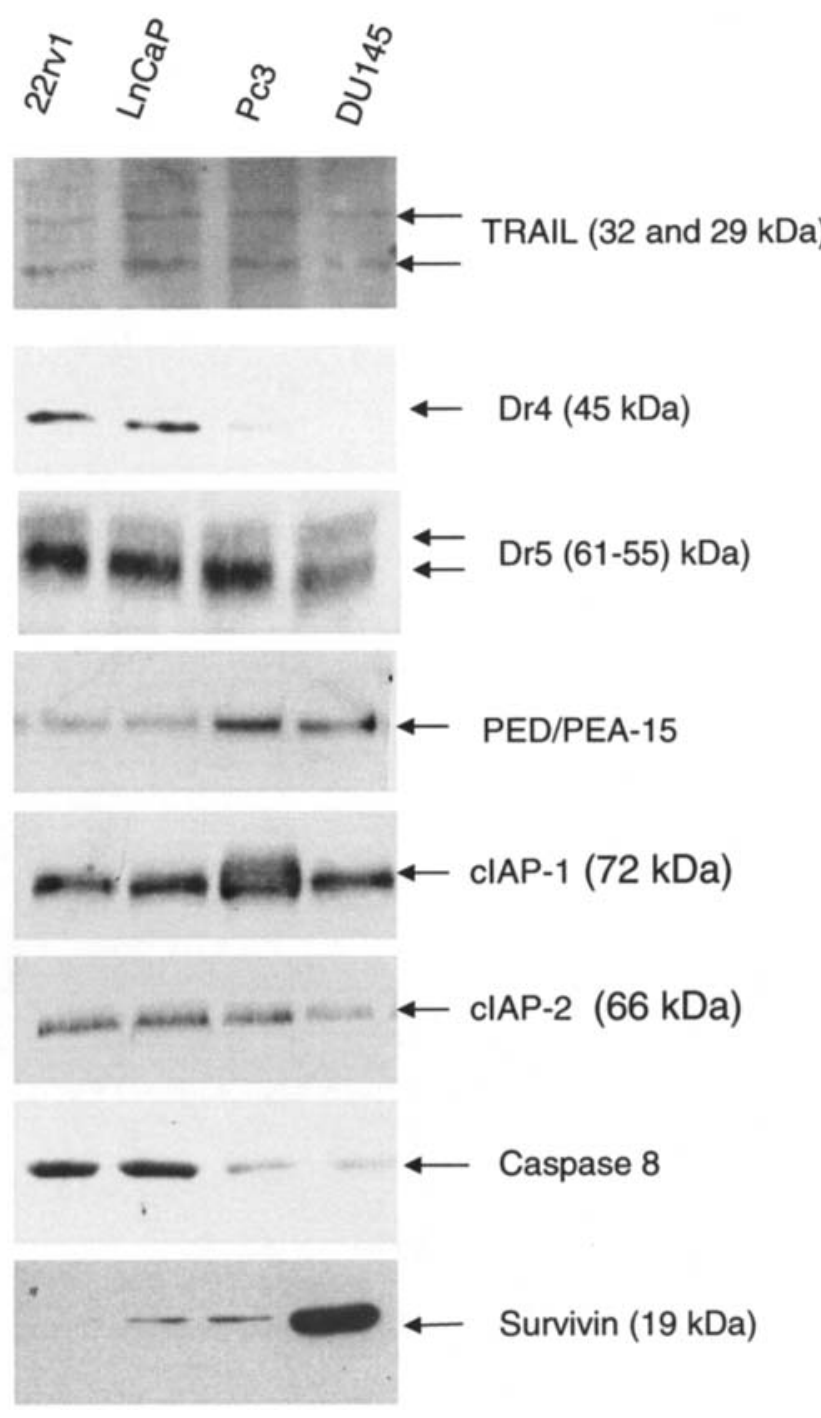

Figure 4. Molecular arrangements involved in TRAIL-dependent cell death in four PCa cells lines having different TRAIL effectiveness. dependent results were observed for 22rv1 cells in which even $0.1 \mu \mathrm{M}$ decitabine shows a significant modification in the analyzed molecular mediators of apoptosis. Analysis of p53-mediated $\mathrm{p} 21$ induction and $\mathrm{Bcl}-2$ reduction also revealed that tumor cells differing in p53 status show corresponding differences in sensitivity to decitabine toxicity. In fact, decitabine-induced cell-arrest/apoptosis was higher in p53 wt LnCaP and 22rv1 cells than in p53 negative PC3 and p53 mutant inactive DU145 cells. In addition, we observed that in PC3 and DU145 cells, caspase- 8 and -9 expression levels were very low in basal conditions and that these increased after decitabine treatment. However, this caspase induction was not able to produce appreciable cell death without $\mathrm{p} 21$ induction or Bcl2 downmodulation (data not shown). We observed that these cells were induced to modulate MAPK pathways. Indeed, Erk activity was reduced and p38 MAPK activity was induced. Fig. 2B shows the molecular variations in DU145 cells treated with high doses of decitabine 10,25 and $50 \mu \mathrm{M}$ corresponding to $\mathrm{IC}_{20}, \mathrm{IC}_{50}$ and $\mathrm{IC}_{80}$ values, respectively. The increased activity of p38 MAPK could be required for the induction of cell cycle arrest in the G2 phase. The induction of both caspase- 8 and -9 also suggests that the apoptotic process can be modulated by the activation of death receptors which are present but not activated in well- and poorly-differentiated PCa cells. Therefore, we investigated the possible role of decitabine in restoring cell sensitivity to the tumor necrosis factor-related apoptosisinducing ligand TRAIL. As expected, human recombinant TRAIL $10-500 \mathrm{ng} / \mathrm{ml}$ induced a modest anti-proliferative effect in PC3 and DU145 cell lines (Fig. 3A) and these did not undergo apoptosis even when exposed to high doses of TRAIL (Fig. 3C); on the contrary, the more differentiated cells 22rv1 particularly and LnCaP less evidently were sensitive to TRAIL-induced cell growth inhibition (Fig. 3A) and apoptosis (Fig. 3C). However, a single 24-h pulse of decitabine, used at relative $\mathrm{IC}_{50}$ values, followed by $72 \mathrm{~h}$ of 
A

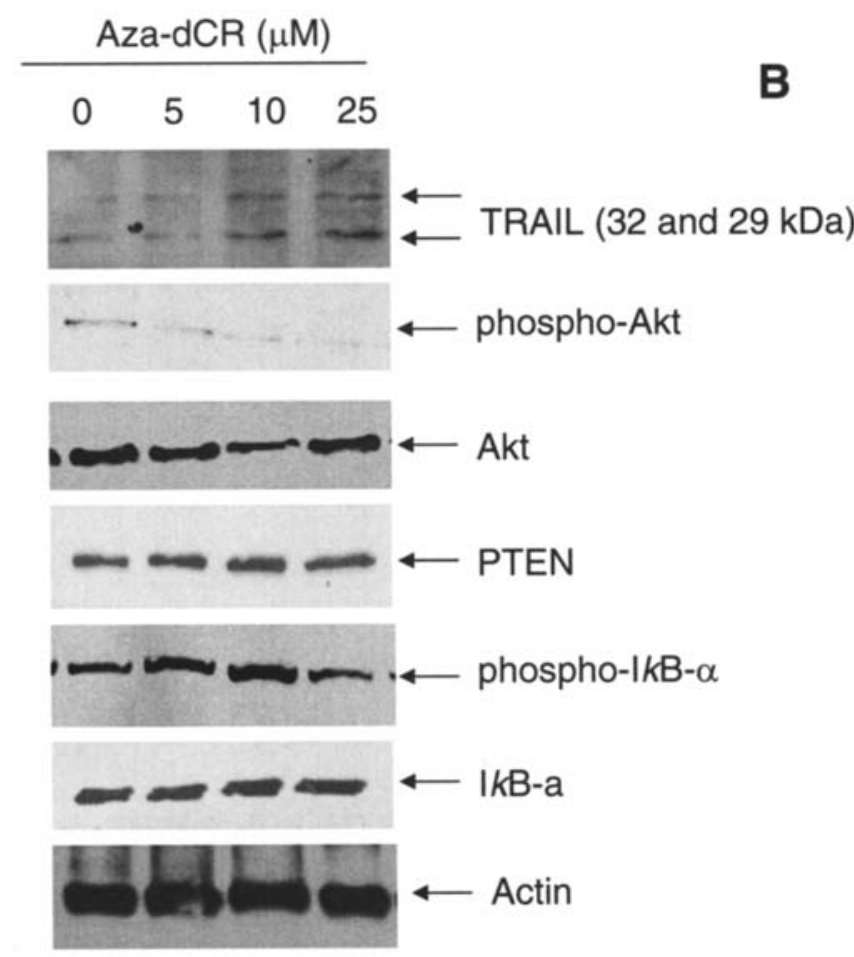

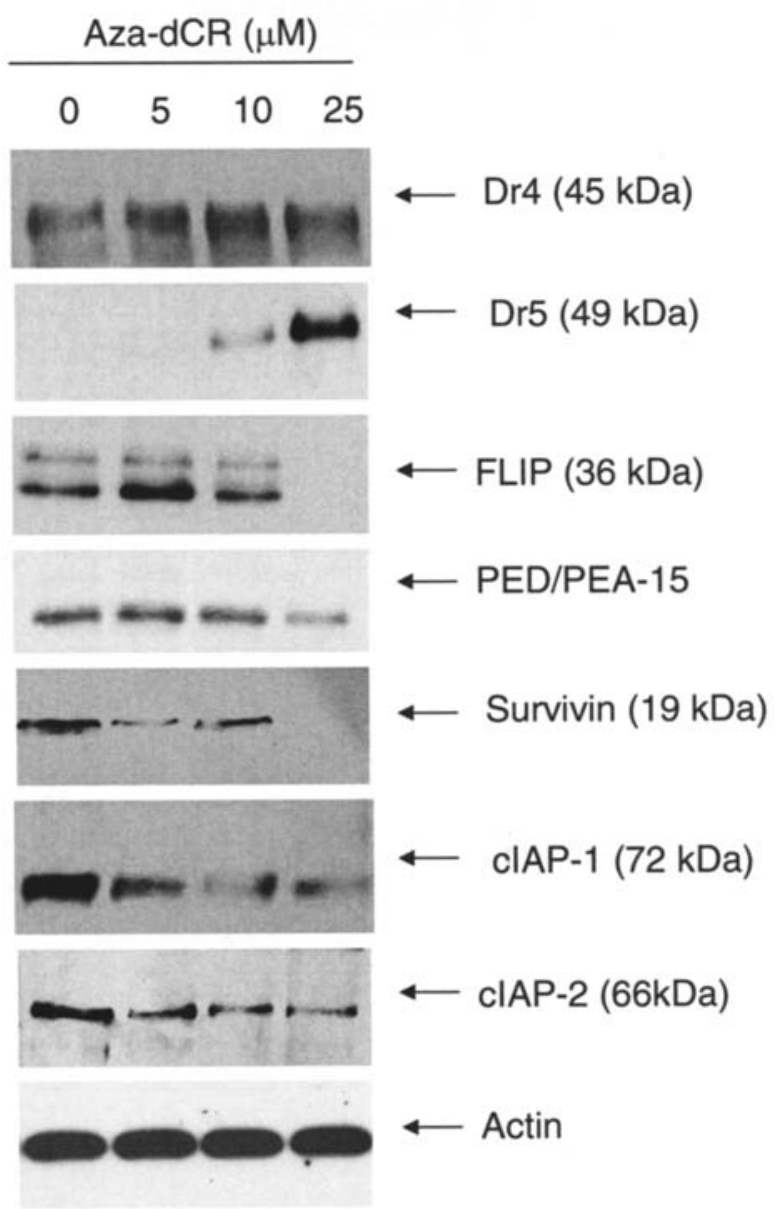

Figure 5. Molecular events involved in TRAIL-depedent cell death after decitabine pretreatment in PC3 cells. Role of the active form of Akt in aza-CR-induced

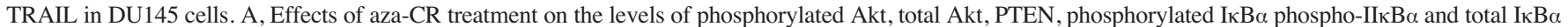
were determined by Western blotting. B-actin was used as a loading control. B, Effects of aza-CR treatment on the levels of death receptors Dr4 and Dr5, antiapoptotic protein FLICE-inhibitory protein FLIP, caspase-8 inhibitory protein PED/PEA-15, Survivin, cIAPs 1 and 2.

culture in drug-free medium, resulted in a considerable dosedependent growth reduction (Fig. 3B) and apoptosis (Fig. 3D) after human recombinant TRAIL was added to all cell models used in the study. However, the effects were higher in undifferentiated PC3 and DU145 cells when compared to differentiated $\mathrm{LnCaP}$ and 22rv1 cells. The comparison of combination indices revealed that decitabine was additive with TRAIL in 22rv1 CI $=0.80$ and $\mathrm{LnCaP} \mathrm{CI}=0.91$ cells and was super-additive synergistically in PC3 CI $=0.35$ and DU145 CI $=0.27$ cells.

To explore the mechanisms responsible for TRAIL resistance, we investigated whether an altered expression of key elements of the proximal TRAIL pathway could be involved in impaired transmission of the apoptotic signal. We first analyzed the expression levels of TRAIL and TRAIL receptors death receptors DR4, TRAIL-R1 and DR5, TRAIL-R2 in our cell system. We observed that all cells secreted appreciable amounts of TRAIL whereas DR4 and DR5 levels were very low and very high, respectively, in all the cells analyzed. Fig. 4 shows that caspase- 8 inhibitory protein PED/PEA15 expression was expressed at similar levels in 22rv1 and LnCaP cells whereas these were higher in PC3 and DU145 cells. Moreover, the levels of cIAP-1 was similar and low in 22rv1, LnCaP and DU145 cells and considerably higher in PC3 cells. The levels of cIAP-2 was similar and low in 22rv1, LnCaP and PC3 cells and considerably lower in DU145 cells. Survivin was absent in 22rv1, low in $\mathrm{LnCaP}$ and PC3 and very high in DU145 cells. Levels of caspase-8 were very low in PC3 and DU145 cells, when compared to 22rv1 and LnCaP cells.

We observed that decitabine treatment was not able to increase the levels of secreted TRAIL in our cell line panel. Moreover, decitabine treatment was able to reduce akt activation. Fig. 5A shows the effects of decitabine treatment in DU145 cells. Death receptor expression analysis of DR4 and DR5 revealed that aza-CR induced these proteins (Fig. 5B) in DU145 cells. Similar results were obtained in PC3 and to a lesser extent in LnCaP and 22rv1 cells. Contemporaneously, protective molecules against TRAIL-mediated cell apoptosis like FLIP, PEA15, survivin and cIAP-1, but not cIAP-2 were significantly reduced. Fig. 5B shows the effects of decitabine treatment on FLIP, PEA15 and c-IAPs expression in DU145 cells. When we combined decitabine and TRAIL we observed that decitabine was able to synergize with TRAIL inducting a strong cell death in TRAIL-resistant PC3 and DU145 cells, in a dose-dependent manner, by activating caspase- 8 and -9 and by cleaving PARP (Fig. 6). 
A

B
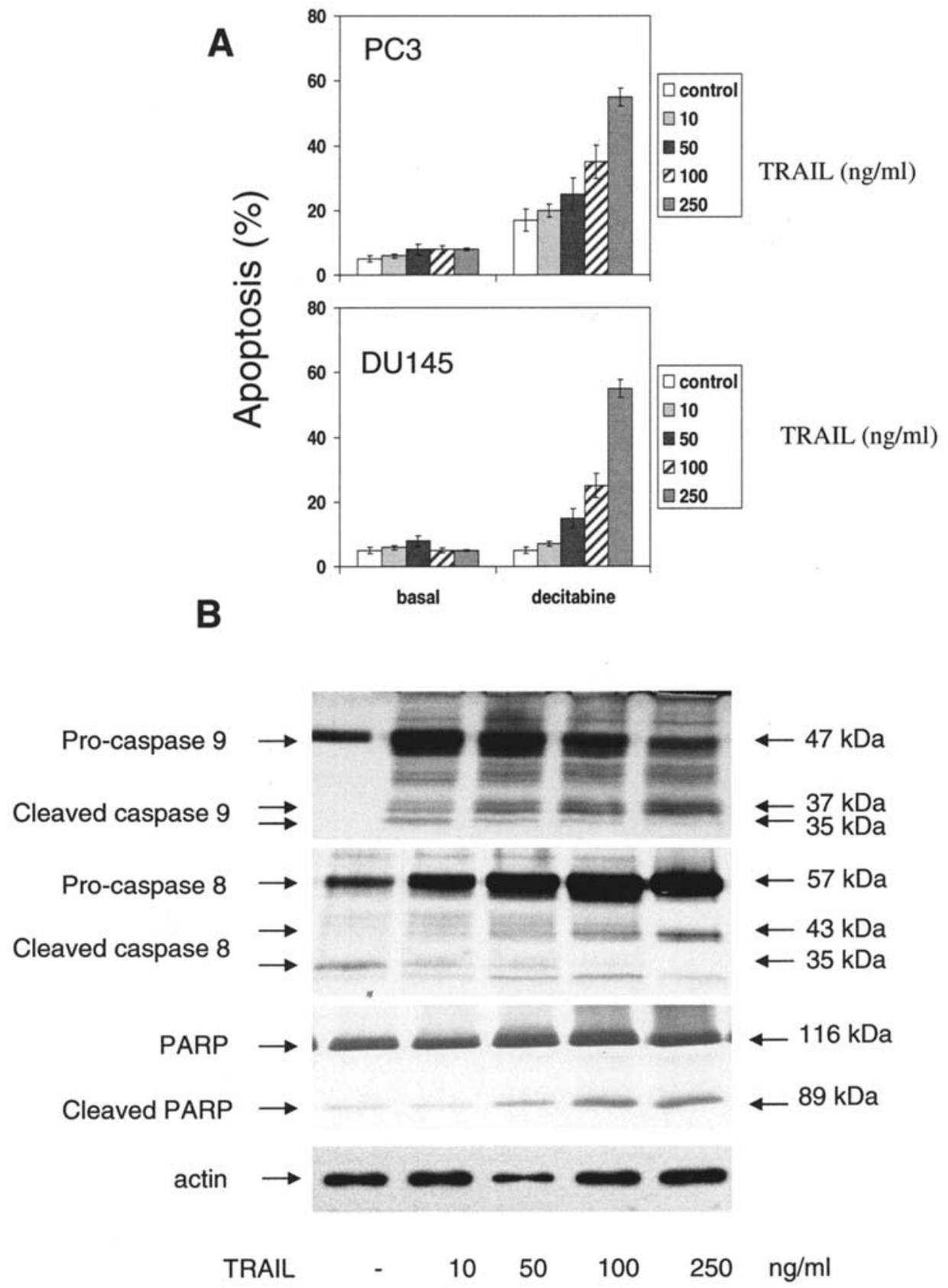

Figure 6. A, TRAIL-depedent cell death after decitabine pretreatment in PC3 and DU145 cells cells. B, Caspase activation and PARP cleavage in decitabine pretreated PC3 cells by TRAIL.

\section{Discussion}

Abnormal DNA methylation patterns observed in cancer cells usually correspond to overall hypomethylation of the genome accompanied by regional hypermethylation. Treatment of PCa cell lines with decitabine caused p53 induction and a dose- and time-dependent inhibition of cell proliferation. We found that wild-type p53-expressing $\mathrm{LnCaP}$ cells are more sensitive to decitabine-induced toxicity than p53 defective PC3 and DU145 cell lines. Therefore, we examined whether cellular toxicity and p53 activation after decitabine treatment was a consequence of specific DNA methyltransferase inhibition. PC3 and DU145 cells p53 null were induced to a G2/M cell cycle arrest with increased activation of p38 MAPK without downmodulation of Akt activity and induction of Bad and p21 activity which are triggered in a normal program of cell death apoptosis. Although tumor necrosis factor-related apoptosis-inducing ligand TRAIL is potentially an excellent and selective therapeutic agent with minimal toxic side-effects, PCa cells develop resistance to TRAIL. We showed that PCa cells are completely refractory to TRAILmediated apoptosis. However, treatment with decitabine restored TRAIL responsiveness through caspase- 8 and TRAIL-R1 up-regulation and through PED/PEA-15 downregulation. We observed that high levels of the anti-apoptotic protein PED/PEA-15 correlated with increased resistance to TRAIL. The balance between the expression of caspase- 8 and its inhibitor PED/PEA-15 seems extremely relevant for determining susceptibility to TRAIL-mediated apoptosis. DNA methylation inhibition in $\mathrm{PCa}$ cells resulted in a 
modification of this balance in favour of caspase- 8 , thus increasing apoptosis susceptibility. Although methyltransferase-mediated caspase- 8 silencing occurs in other cancers, there is a lack of agreement concerning the identification of the promoter responsible for epigenetic regulation of caspase- 8 expression. Therefore, the significance of direct caspase- 8 promoter demethylation or transacting factors acting on this promoter in decitabine-induced caspase- 8 up-regulation remains to be determined. We also found that DNA demethylation resulted in increased TRAIL-R1 expression. Decitabine-induced TRAIL-R1 expression could represent a possible sensitization strategy to treat these types of cancer. For instance, low levels of TRAIL-R1 expression seem to be associated with TRAIL resistance in non-small cell lung carcinoma cells. A combined treatment with decitabine and IFN- $\gamma$ was reported to increase both TRAIL-R1 levels and apoptosis sensitivity of these cells, indicating that epigenetic control of TRAIL-R1 transcription might occur in some other cancers (22). Decitabine has been used in humans for the treatment of myelodysplastic syndromes, leukemia, and solid tumors. Phase I and II trials showed that decitabine is well tolerated and moderately effective in some types of cancer (26-29). To date, no data are available concerning clinical toxicity of TRAIL and agonist TRAIL receptor antibodies, currently undergoing phase I and II studies. However, our experimental data are very promising with respect to antitumor activity high and toxicity low. However, intense efforts are essential in order to assess the possible clinical use of decitabine and TRAIL combinations for the treatment of advanced forms of PCa given the high malignancy of these tumors and the short life expectancy in these patients.

\section{References}

1. Jemal A, Siegel R, Ward E, Murray T, Xu J and Thun MJ: Cancer statistics, 2007. CA Cancer J Clin 57: 43-66, 2007.

2. Salesi N, Carlini P, Ruggeri EM, et al: Prostate cancer: the role of hormonal therapy. J Exp Clin Cancer Res 24: 175-180, 2005.

3. Arnold JT and Isaacs JT: Mechanisms involved in the progression of androgen-independent prostate cancers: it is not only the cancer cell's fault. Endocr Relat Cancer 9: 61-73, 2002.

4. Ai L, Kim WJ, Kim TY, Fields CR, Massoll NA, Robertson KD and Brown KD: Epigenetic silencing of the tumor suppressor cystatin $\mathrm{M}$ occurs during breast cancer progression. Cancer Res 66: 7899-7909, 2006.

5. Lodygin D, Epanchintsev A, Menssen A, Diebold J and Hermeking $\mathrm{H}$ : Functional epigenomics identifies genes frequently silenced in prostate cancer. Cancer Res 65: 42184227,2005

6. Cho NY, Kim BH, Choi M, et al: Hypermethylation of $\mathrm{CpG}$ island loci and hypomethylation of LINE-1 and Alu repeats in prostate adenocarcinoma and their relationship to clinicopathological features. J Pathol 211: 269-277, 2007.

7. Karpf AR, Moore BC, Ririe TO and Jones DA: Activation of the p53 DNA damage response pathway after inhibition of DNA methyltransferase by 5-aza-2'-deoxycytidine. Mol Pharmacol 59: 751-757, 2001.

8. Pulukuri SM and Rao JS: Activation of p53/p21Waf1/Cip1 pathway by 5 -aza-2'-deoxycytidine inhibits cell proliferation, induces pro-apoptotic genes and mitogen-activated protein kinases in human prostate cancer cells. Int J Oncol 26: 863-871, 2005.

9. Momparler RL, Bouchard J and Samson J: Induction of differentiation and inhibition of DNA methylation in HL-60 myeloid leukemic cells by 5-AZA-2'-deoxycytidine. Leuk Res 9: 1361-1366, 1985.
10. McCabe MT, Low JA, Daignault S, Imperiale MJ, Wojno KJ and Day ML: Inhibition of DNA methyltransferase activity prevents tumorigenesis in a mouse model of prostate cancer. Cancer Res 66: 385-392, 2006.

11. Shresta S, Pham CT, Thomas TA Graubert and Ley TJ: How do cytotoxic lymphocytes kill their targets? Curr Opin Immunol 98: 581-587, 1998.

12. Thompson CB: Apoptosis in the pathogenesis and treatment of disease. Science 267: 1456-1462, 1995.

13. Ng CP and Bonavida B: A new challenge to immunotherapy by tumors that are resistant to apoptosis: two complementary signals to overcome cross-resistance. Adv Cancer Res 85: 145-174, 2002.

14. Yamana K, Bilim V, Hara N, et al: Prognostic impact of FAS/CD95/APO-1 in urothelial cancers: decreased expression of Fas is associated with disease progression. $\mathrm{Br} \mathrm{J}$ Cancer 93: 544-551, 2005.

15. Irmler M, Thome M, Hahne MP, et al: Inhibition of death receptor signals by cellular FLIP. Nature 388: 190-195, 1997.

16. Roth W, Isenmann S, Nakamura M, et al: Soluble decoy receptor 3 is expressed by malignant gliomas and suppresses CD95 ligand-induced apoptosis and chemotaxis. Cancer Res 61: 2759-2765, 2001

17. Kim S, Kang J, Qiao J, Thomas RP, Evers BM and Chung DH: Phosphatidylinositol 3-kinase inhibition down-regulates survivin and facilitates TRAIL-mediated apoptosis in neuroblastomas. J Pediatr Surg 39: 516-521, 2004.

18. Larribere L, Khaled M, Tartare-Deckert S, et al: PI3K mediates protection against TRAIL-induced apoptosis in primary human melanocytes. Cell Death Differ 11: 1084-1091, 2004.

19. Rokhlin OW, Taghiyev AF, Guseva NV, Glover RA, Syrbu SI and Cohen MB: TRAIL-DISC formation is androgen-dependent in the human prostatic carcinoma cell line $\mathrm{LNCaP}$. Cancer Biol Ther 1: 631-637, 2002.

20. Harada K, Toyooka S, Shivapurkar N, et al: Deregulation of caspase 8 and 10 expression in pediatric tumors and cell lines. Cancer Res 62: 5897-5901, 2002.

21. Eramo A, Pallini R, Lotti F, et al: Inhibition of DNA methylation sensitizes glioblastoma for tumor necrosis factor-related apoptosis-inducing ligand-mediated destruction. Cancer Res 65: 11469-11477, 2005.

22. Eggert A, Grotzer MA, Zuzak TJ, Wiewrodt BR, Ho R, Ikegaki N and Brodeur GM: Resistance to tumor necrosis factor-related apoptosis-inducing ligand TRAIL-induced apoptosis in neuroblastoma cells correlates with a loss of caspase-8 expression. Cancer Res 61: 1314-1319, 2001.

23. Thakkar $\mathrm{H}$, Chen X, Tyan $\mathrm{F}$, et al: Pro-survival function of Akt/protein kinase B in prostate cancer cells. Relationship with TRAIL resistance. J Biol Chem 276: 38361-38369, 2001.

24. Chen X, Thakkar H, Tyan F, et al: Constitutively active Akt is an important regulator of TRAIL sensitivity in prostate cancer. Oncogene 20: 6073-6083, 2001.

25. Dorothee G, Vergnon I, Menez J, et al: Tumor-infiltrating $\mathrm{CD}^{+} \mathrm{T}$ lymphocytes express APO2 ligand APO2L/TRAIL upon specific stimulation with autologous lung carcinoma cells: role of IFN-alpha on APO2L/TRAIL expression and -mediated cytotoxicity. J Immunol 169: 809-817, 2002.

26. Gollob JA, Sciambi CJ, Peterson BL, et al: Phase I trial of sequential low-dose 5-aza-2'-deoxycytidine plus high-dose intravenous bolus interleukin-2 in patients with melanoma or renal cell carcinoma. Clin Cancer Res 12: 4619-4627, 2006.

27. Pohlmann P, Di Leone LP, Cancella AI, et al: Phase II trial of cisplatin plus decitabine, a new DNA hypomethylating agent, in patients with advanced squamous cell carcinoma of the cervix. Am J Clin Oncol 25: 496-501, 2002.

28. Schrump DS, Fischette MR, Nguyen DM, et al: Phase I study of decitabine-mediated gene expression in patients with cancers involving the lungs, esophagus, or pleura. Clin Cancer Res 12: 5777-5785, 2006.

29. Tolcher AW, Mita M, Meropol NJ, et al: Phase I pharmacokinetic and biologic correlative study of mapatumumab, a fully human monoclonal antibody with agonist activity to tumor necrosis factor-related apoptosis-inducing ligand receptor-1. J Clin Oncol 25: 1390-1395, 2007. 